Check for updates

Cite this: RSC Adv., 2017, 7, 44673

\section{The effect of hydrophobic alkyl chain length on the mechanical properties of latex particle hydrogels}

\author{
Yang Gao, ${ }^{a}$ Lijie Duan, ${ }^{b}$ Shuang Guan, ${ }^{b}$ Guanghui Gao, (D) a Ya Cheng, ${ }^{a}$ \\ Xiuyan Ren (D) *a and Yuanrui Wang*a
}

Herein, different long alkyl chains (C1, C6, C12, and C16) were introduced as hydrophobic segments to enhance the performance of hydrogels reinforced by latex particles (LP-Gel). Poly(butyl acrylate) (PBA) latex particles (LPs) were employed as hydrophobic association cross-linking centers. First, the PBA latex particles were prepared via emulsion polymerization, and then, LP-Gel with high mechanical strength was prepared via one-pot free radical polymerization using acrylamide as a monomer, LP as a crosslinking center, and methacrylate as a hydrophobic molecule. It was found that the length of the hydrophobic alkyl chains from methacrylate has a significant effect on the mechanical performance and swelling degree of the hydrogels. The short alkyl chains exhibited weak hydrophobic interactions, and the resulting LP-Gel had a low mechanical strength. However, the long alkyl chains can effectively entangle with LPs through strong hydrophobic interactions, which significantly enhance the mechanical strength of the hydrogels. As a result, the LP-Gel exhibits a maximum fracture stress of $1.2 \mathrm{MPa}$ and elongation of $2336 \%$. This study will have a profound impact on the understanding of hydrogels toughened by hydrophobic alkyl chains of different lengths.
Received 20th July 2017

Accepted 4th September 2017

DOI: $10.1039 / \mathrm{c} 7 \mathrm{ra0} 0798 \mathrm{~d}$

rsc.li/rsc-advances alcohol polyoxyethylene acrylate molecules. The mechanical strength could be tuned by adjusting the ratio of monomers and the concentration of surfactants. However, the maximum tensile strength was $318 \mathrm{kPa}{ }^{31}$ Wang and co-workers successfully fabricated hydrophobically associated hydrogels based on acrylamide and anionic surfactant monomers. The anionic surfactant segment formed multi-micellar aggregates and acted as cross-linking centers to endow the hydrogels with high mechanical strength. ${ }^{32}$ Furthermore, a series of double-network hydrogels and hybrid hydrogels was fabricated by introducing hydrophobic segments. Chen et al. designed a novel doublenetwork physical cross-linking hydrogel. The hydrophobic association polyacrylamide hydrogel acted as a second network to effectively dissipate energy and improve strength. ${ }^{16}$ Moreover, Cui and co-workers constructed a hybrid hydrogel cross-linked by graphene oxide sheets and hydrophobically associated domains that exhibited anti-fatigue and self-healing properties. However, the fracture stress of the hydrogel was only $260 \mathrm{kPa} .^{33}$

In our previous study, a novel method was proposed to improve the mechanical properties of hydrogels via the introduction of latex particles (LPs) as hydrophobic association cross-linking centers. Hexadecyl methacrylate (HMA) as a hydrophobic monomer could be adsorbed on the surface of the LPs due to hydrophobic interactions; this forms the LPGel. ${ }^{34}$ However, the length of the hydrophobic alkyl chains could not be considered to enhance the mechanical strength of hydrogels. We considered that the hydrophobic segments could exert a great influence on the interaction between the
${ }^{a}$ School of Chemical Engineering, Changchun University of Techn
130012, China. E-mail: xyren_bio@163.com; wyr@ccut.edu.cn

${ }^{b}$ School of Chemistry and Life Science, Changchun University of Technology, Changchun 130012, China 
hydrophobic segments and latex particles and affect the mechanical behavior of the LP-Gel. Therefore, it was crucial to study the effects of different long hydrophobic chains on the mechanical properties based on the system of latex particles toughening hydrogels.

Herein, we introduced different hydrophobic monomers to enhance the mechanical strength of hydrogels reinforced by LPs as cross-linking centers for hydrophobic association. The mechanical performance of hydrogels can be measured by tuning the contents of LPs and the length of hydrophobic alkyl chains. Subsequently, the swelling behavior and rheological performance of the hydrogels were also investigated by varying the length of the hydrophobic alkyl chains. These results are meaningful for the design of a new generation of novel latex particle hydrogels.

\section{Experimental}

\section{Materials}

Acrylamide (AAm; 99.0\%), potassium persulphate (KPS; 99.5\%), sodium dodecyl sulfate (SDS; 97\%), $N, N, N^{\prime}, N^{\prime}$-tetramethylethylenediamine (TMEDA; 99.5\%), methyl methacrylate (MMA; 99.5\%), hexyl methacrylate (HMA; 98\%), lauryl methacrylate (LMA; 96\%), isocyanuric acid triallyl ester (TAIC; 98\%), and sodium carbonate $\left(\mathrm{Na}_{2} \mathrm{CO}_{3} ; 99.0 \%\right)$ were supplied by Aladdin. Hexadecyl methacrylate (HDMA; 95\%) was purchased from Zhejiang Kangde New Materials Co. Ltd., China. MMA and HMA were distilled under reduced pressure, and LMA and HDMA were passed three times through a column packed with basic alumina. Butyl acrylate (BA) was bought from Aladdin and further purified by distillation under reduced pressure. Sodium chloride ( $\mathrm{NaCl}$; 99.5\%) was obtained from Beijing Chemical Factory. Deionized water was used in all the experiments.

\section{Preparation of PBA LMs}

SDS $(0.4 \mathrm{~g})$ and $\mathrm{Na}_{2} \mathrm{CO}_{3}(0.24 \mathrm{~g})$ were dissolved in deionized water $(35 \mathrm{~mL})$ in a three-necked boiling flask. Subsequently, BA $(60 \mathrm{~g})$ and TAIC $(0.3 \mathrm{~g})$ were added to the reactor and stirred for 30 min under the protection of oxygen gas. Finally, a certain amount of KPS $(0.24 \mathrm{~g})$ was dissolved in $5 \mathrm{~mL}$ deionized water, and the solution was added to the reactor. The reaction was carried out at $65 \pm 1{ }^{\circ} \mathrm{C}$ for $2 \mathrm{~h}$ to achieve high conversation rate. The obtained yield was $97.4 \%$. Finally, the PBA LPs were obtained and could be utilized directly in the following process.

\section{Preparation of hydrogels}

The hydrogels were synthesized by free radical polymerization of AAm and different hydrophobic monomers in the presence of the abovementioned latex particles (LPs) as cross-linking centers for hydrophobic association. For example, the P(AAm/ LMA)-LP hydrogel was prepared as follows: SDS $(0.21 \mathrm{~g})$ and $\mathrm{NaCl}(0.168 \mathrm{~g})$ were dissolved in deionized water $(27 \mathrm{~mL})$ under stirring at $25{ }^{\circ} \mathrm{C}$ for $15 \mathrm{~min}$ to obtain a transparent solution. LMA (0.07 g) and LPs $(0.0175,0.035,0.07$, and $0.105 \mathrm{~g}$ ) were added to the abovementioned SDS-NaCl solution under stirring for $6 \mathrm{~h}$ to form a homogeneous solution. AAm $(7 \mathrm{~g})$ and $1 \mathrm{~mL}$ of initiator KPS $\left(0.03 \mathrm{~g} \mathrm{~mL}^{-1}\right)$ were added and stirred for $10 \mathrm{~min}$. Subsequently, TMEDA $(60 \mu \mathrm{L})$ was added to the abovementioned uniform solution. Finally, the resulting solution was injected into a mold consisting of a pair of parallel glass plates and a silicone spacer (thickness: $3 \mathrm{~mm}$ ), and polymerization was conducted at $30^{\circ} \mathrm{C}$ for $12 \mathrm{~h}$. For comparison, the P(AAm/ LMA) hydrogel and PAAm-LP hydrogel were fabricated according to the abovementioned procedure. P(AAm/MMA)-LP, $\mathrm{P}(\mathrm{AAm} / \mathrm{HMA})-\mathrm{LP}$, and P(AAm/HDMA)-LP hydrogels were also obtained by the same experimental process. The mole ratio of hydrophobic monomer was fixed at $0.28 \mathrm{~mol} \%$ of AAm.

\section{Dynamic light scattering measurements}

The dynamic light scattering (American Brookhaven 90Plus Particle Size Analyzer) experiments were performed on the LPS and the mixed solution of LPs and hydrophobic monomers (MMA, HMA, LMA, and HDMA) to characterize their particle size and size distribution. The laser light-scattering angle was fixed at $90^{\circ}$. The samples were diluted in deionized water with a concentration of $0.0002 \mathrm{mg} \mathrm{mL} \mathrm{m}^{-1}$ for analysis. Each sample was tested at least five times, and the average particle size was obtained.

\section{Mechanical tests}

The mechanical tests were performed on the as-prepared samples using a tensile tester (SHIMADZU, model AGS-X, $100 \mathrm{~N}$, Japan) at room temperature. The hydrogel specimens were cut into a dumbbell shape with a gauge length of $30 \mathrm{~mm}$, width of $4 \mathrm{~mm}$, and a depth of $3 \mathrm{~mm}$. The crosshead speed used in all the experiments was fixed at $100 \mathrm{~mm} \mathrm{~min}^{-1}$. The value of toughness was obtained by calculating the area below the stress-strain curve of each sample, whereas the elastic modulus was determined by fitting the initial linear region (between $0 \%$ and $20 \%$ for elongation) of the stress-strain curve. To ensure accuracy, five specimens of each sample were tested, and the average values were obtained. For the cyclic tensile experiments, the samples were first loaded to the strain of $1000 \%$ with a constant velocity of $100 \mathrm{~mm} \mathrm{~min} \mathrm{~m}^{-1}$ and then unloaded at the same velocity. The dissipated energies were estimated by the area between loading-unloading curves.

\section{Rheological measurements}

The rheological measurements of the hydrogels were carried out using a TA AR 2000ex rheometer in the oscillatory mode at $25{ }^{\circ} \mathrm{C}$. The measuring device was equipped with a parallel plate configuration $(25 \mathrm{~mm}$ in diameter and $2 \mathrm{~mm}$ between gap distance). The storage modulus $G^{\prime}$ and loss modulus $G^{\prime \prime}$ were subjected to frequency sweeping tests in the angular frequency range from 0.1 to $100 \mathrm{rad} \mathrm{s}^{-1}$. The gelation reactions of the four different hydrogels were monitored by the rheometer at $30{ }^{\circ} \mathrm{C}$ up to a reaction time of about $40 \mathrm{~min}$.

\section{Swelling behavior}

The hydrogel samples prepared using different hydrophobic monomers (MMA, HMA, LMA, and HDMA) with a thickness of 
$2 \mathrm{~mm}$ and a diameter of $15 \mathrm{~mm}$ were dried in a vacuum oven at $60{ }^{\circ} \mathrm{C}$ for $24 \mathrm{~h}$ to a constant weight. The dried hydrogels were then immersed in an excess of deionized water $(50 \mathrm{~mL})$. Deionized water was changed regularly during the test. Swollen hydrogels were periodically removed from deionized water and weighed after blotting the surface water with filter paper. The swelling ratio was calculated by the following equation:

$$
\mathrm{SR}=\left(\left(W_{\mathrm{s}}-W_{\mathrm{d}}\right) / W_{\mathrm{d}}\right)
$$

where $W_{\mathrm{s}}$ is the weight of the swollen hydrogel and $W_{\mathrm{d}}$ is the weight of the dried hydrogel. Each sample was tested thrice, and the average values were calculated.

\section{Morphological observation}

The hydrogel morphology was observed by scanning electron microscopy (SEM, JSM6510, JEOL). The samples were freezedried under vacuum using a freeze dryer (FDU-2110, Eyela). Before the measurement, all the samples were broken by inserting into liquid nitrogen for $3 \mathrm{~min}$ and then sputtered with platinum. The magnification factor was 200 times.

\section{Results and discussion}

In this investigation, poly(butyl acrylate) (PBA) latex particles (LPs) were designed as association cross-linking centers for hydrophobic segments to improve the mechanical strength of the hydrogels. PBA LPs were synthesized by an emulsion polymerization method using TAIC as a cross-linker and KPS as an initiator. The particle size and size distribution of the PBA LPs were characterized by DLS. As shown in Fig. 1, the mean diameter was $293.2 \mathrm{~nm}$, and the size distribution was 0.005 . The particle size of LPs upon absorption of hydrophobic methacrylate was also measured by DLS. As expected, the DLS results indicated that the particle size of the LP-adsorbed hydrophobic monomer presented monodisperse distribution. In other words, the methacrylates (MMA, HMA, LMA, and HDMA) could be adsorbed onto LPs. Simultaneously, the mean diameter

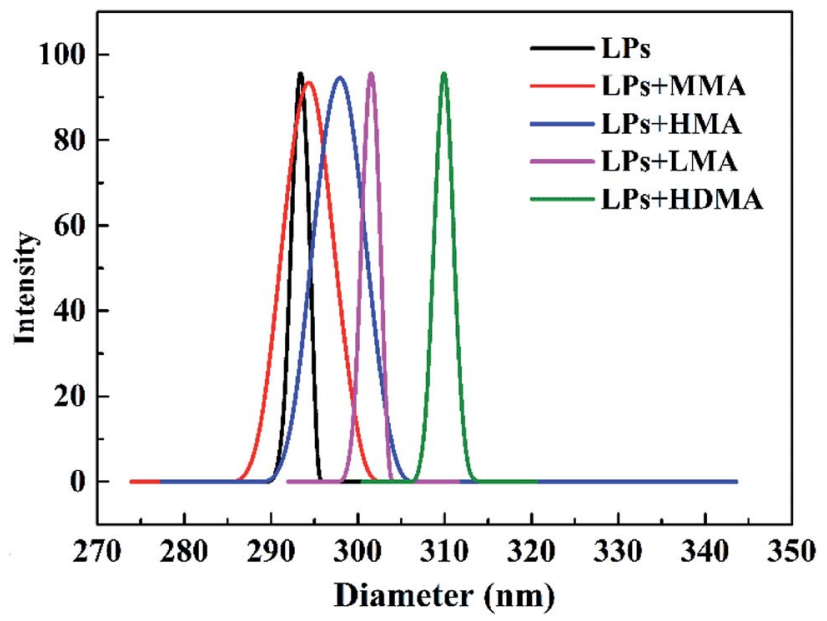

Fig. 1 The particle size of PBA LPs and methacrylate-absorbed LPs. gradually increased with the increase of the hydrophobic alkyl length. The mean diameters of the LPs with MMA, HMA, LMA, and HDMA were 294.2, 297.8, 301.4, and $309.9 \mathrm{~nm}$, respectively.

The hydrogels were synthesized by the radical polymerization of AAm and methacrylates with different length of hydrophobic chains in the presence of the PBA LPs. As illustrated in Fig. 2, the hydrogels were fabricated with LPs as association cross-linking centers for hydrophobic monomers. As a result, four samples, i.e. P(AAm/MMA)-LPs, P(AAm/HMA)-LPs, P(AAm/ LMA)-LPs, and P(AAm/HDMA)-LPs, with different hydrophobic chains were obtained.

For observing the validity of the LP-reinforced hydrogels, first, the typical tensile mechanical performance of PAAm-LP, $\mathrm{P}(\mathrm{AAm} / \mathrm{LMA})$, and P(AAm/LMA)-LP hydrogels was determined (Fig. 3). The tensile strength, toughness, and elastic modulus of the PAAm-LPs hydrogels were only $19 \mathrm{kPa}, 0.41 \mathrm{MJ} \mathrm{m}^{-3}$, and 25 $\mathrm{kPa}$, respectively. Moreover, the related values of P(AAm/LMA) hydrogels were obtained, which were only $560 \mathrm{kPa}, 3.26 \mathrm{MJ}$ $\mathrm{m}^{-3}$, and $40 \mathrm{kPa}$. In contrast, when the LPs were introduced into the $\mathrm{P}(\mathrm{AAm} / \mathrm{LMA})$ hydrogel as association cross-linking centers for hydrophobic segments, the P(AAm/LMA)-LP hydrogels exhibited excellent mechanical performance. The tensile strength, toughness, and elastic modulus of the hydrogels were 1.2 $\mathrm{MPa}, 8.26 \mathrm{MJ} \mathrm{m}^{-3}$, and $50 \mathrm{kPa}$, respectively, much higher than those of the PAAm-LPs and P(AAm/LMA) hydrogels; this suggested that the introduction of the LPs could significantly improve the mechanical performance of the hydrophobic association hydrogels.

Subsequently, the influence of LP content on the mechanical properties of the $\mathrm{P}(\mathrm{AAm} / \mathrm{LMA})-\mathrm{LP}$ hydrogels was systematically investigated. As observed from Fig. 4, the tensile strength and toughness of $\mathrm{P}(\mathrm{AAm} / \mathrm{LMA})-\mathrm{LPs}$ obviously increased and then decreased when the LP concentration varied from 0.25 to $1.5 \mathrm{wt} \%$ for AAm. When the LP concentration for AAm was $0.5 \mathrm{wt} \%$, the tensile strength and toughness of the P(AAm/LMA)LP hydrogel became 1.2 MPa and 8.26 MJ m $\mathrm{m}^{-3}$, respectively. With the increase of the LP concentration, more hydrophobic association cross-linking centers were formed, and the P(AAm/ LMA)-LP hydrogels showed a significant increase of tensile strength and toughness. However, the excess of LPs led to extremely high cross-linking density in the polymer network. The number of cross-linking centers increased and the content

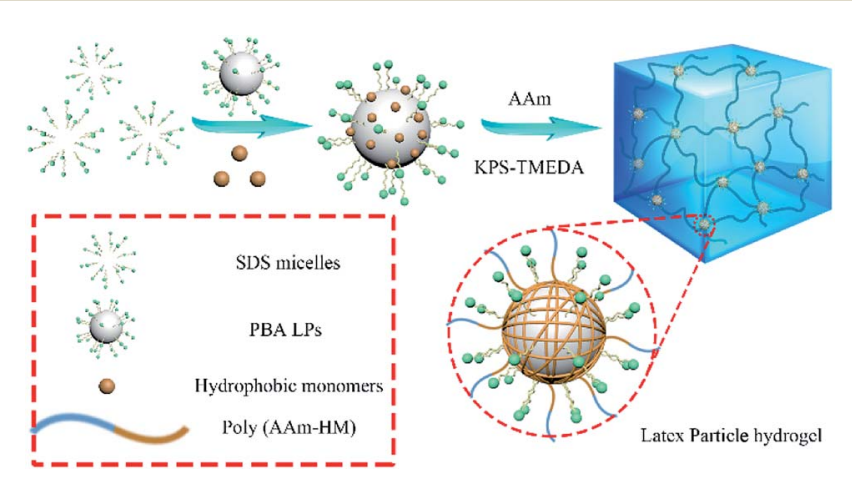

Fig. 2 Schematic of the LP-toughened hydrogels with different hydrophobic monomers. 

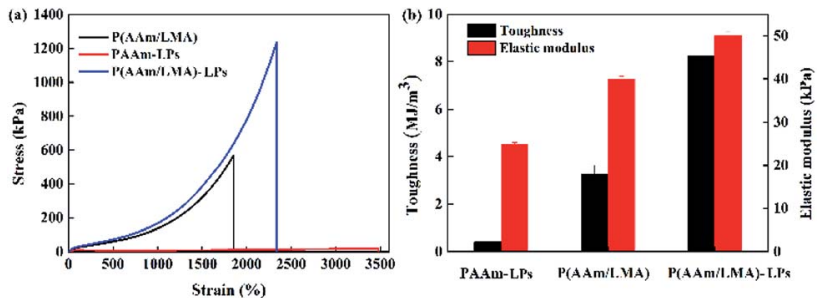

Fig. 3 (a) Tensile stress-strain curves and (b) toughness and elastic modulus of the P(AAm/LMA), PAAm-LPs, and P(AAm/LMA)-LPs hydrogels.
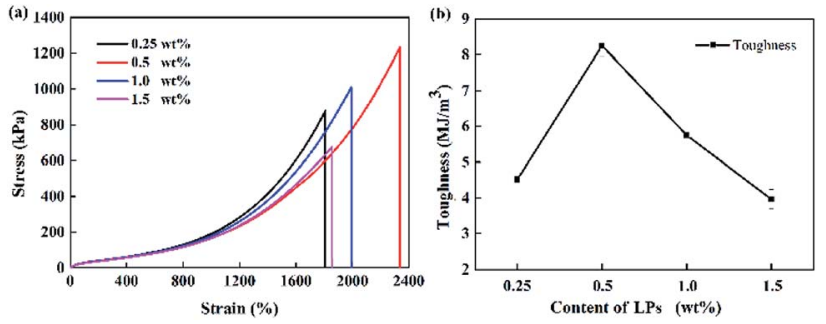

Fig. 4 (a) Tensile stress-strain curves and (b) toughness of P(AAm/ LMA)-LPs hydrogels with different concentrations of LPs.

of hydrophobic monomers adsorbed on each LPs was relatively reduced; this caused the hydrogels to exhibit low strength and fracture elongation. Therefore, an appropriate content of LPs was crucial to enhance the mechanical property of the LP-Gel.

The effect of the hydrophobic alkyl monomers (C1, C6, C12, and C16) on the mechanical properties of the hydrogels was investigated, as illustrated in Fig. 5(a) and (b). It was shown that the hydrogels with MMA and HMA as the hydrophobic
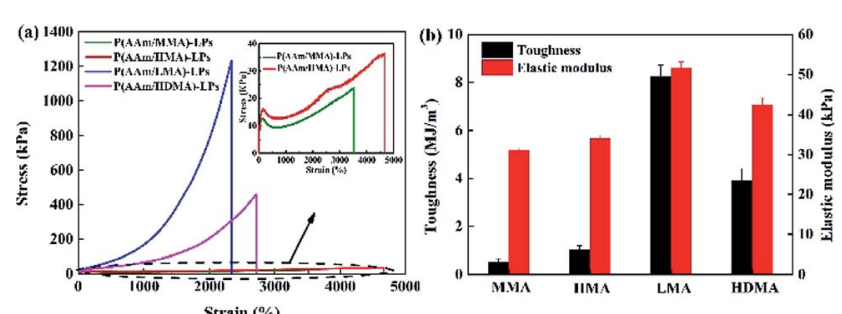

(c)

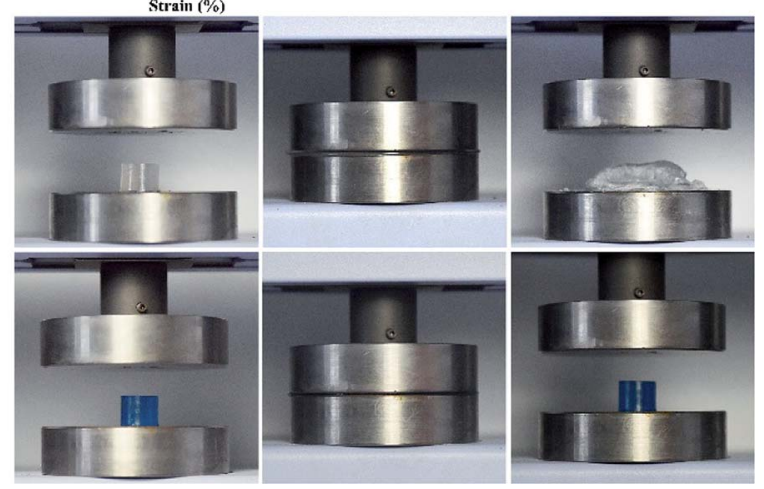

Fig. 5 (a) Tensile stress-strain curves and (b) toughness and elastic modulus of the hydrogels with different hydrophobic segments of MMA, HMA, LMA, and HDMA. (c) The toughness exhibition of P(AAm/ MMA)-LP hydrogels (white) and P(AAm/LMA)-LP hydrogels (blue). monomers exhibited large elongation ratio (3800\% and $4664 \%$ ), but weak tensile strength (23.9 $\mathrm{kPa}$ and $35.4 \mathrm{kPa})$, respectively. However, the hydrogels with LMA and HDMA displayed a moderate elongation ratio (2336\% and $2716 \%$ ) and high tensile strength (1.2 MPa and $471 \mathrm{kPa})$, respectively. Apparently, the tensile strength, toughness, and elastic modulus of the hydrogels considerably increased and then decreased with the increasing alkyl chain length. It is worth noting that the mechanical performance of the P(AAm/LMA)-LP hydrogels is much better than that of the $\mathrm{P}(\mathrm{AAm} / \mathrm{HDMA})-\mathrm{LP}$ hydrogels. Possibly, HDMA with very long alkyl chains was difficult to be absorbed on LPs; this led to a weak cross-linking internal network structure of the hydrogels. As a result, the P(AAm/ HDMA)-LP hydrogels displayed poor mechanical performance as compared to the P(AAm/LMA)-LP hydrogels. To display the toughness of the hydrogels with various alkyl chain length, the $\mathrm{P}(\mathrm{AAm} / \mathrm{MMA})-\mathrm{LP}$ and P(AAm/LMA)-LP hydrogels were subjected to a $90 \%$ strain compressive test. As shown in Fig. 5(c), the $\mathrm{P}(\mathrm{AAm} / \mathrm{MMA})$-LP hydrogel was compressed into a thin slice, whereas the $\mathrm{P}(\mathrm{AAm} / \mathrm{LMA})-\mathrm{LP}$ hydrogel recovered its original length after compression without damage.

The following process was proposed as the toughening mechanism of LP-reinforced hydrogels with different hydrophobic alkyl chains (Fig. 6). For the P(AAm/MMA)-LP and $\mathrm{P}(\mathrm{AAm} / \mathrm{HMA})-\mathrm{LP}$ hydrogels, the short hydrophobic chains were prone to disentangle from LPs under initial deformation due to the weak hydrophobic interaction. The network structure was easy to collapse, resulting in the poor tensile strength of hydrogels. However, the LMA and HDMA hydrophobic segments of hydrogels hardly disentangled from the LPs at the initial deformation. When the external force was applied, the hydrophobic chains would first transform from curling to stretching due to the efficient aggregation of hydrophobic chains on LPs. As the external load increased, the long chains would slide on the surface of LPs to dissipate a large amount of energy.

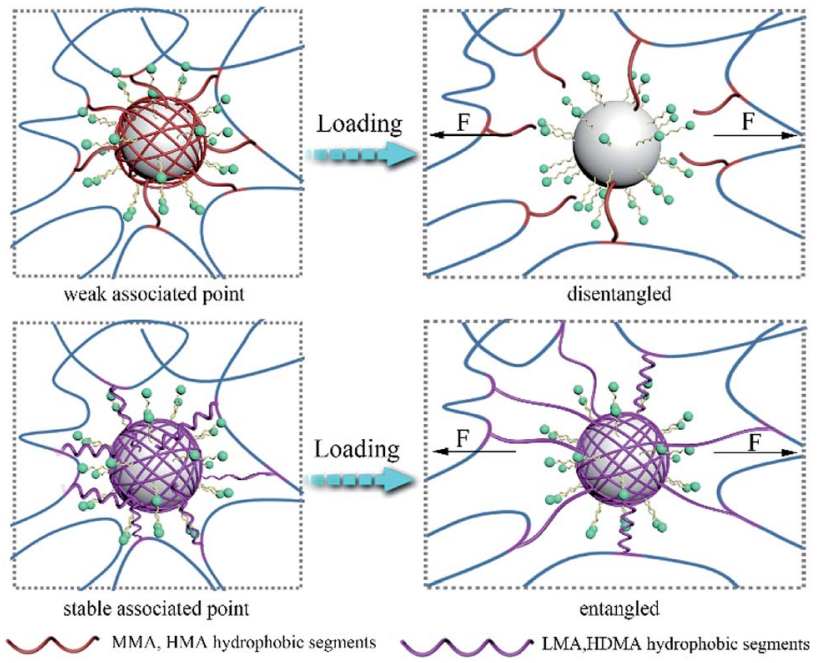

Fig. 6 The toughening mechanism for LP-reinforced hydrogels with different hydrophobic chain lengths. 
Furthermore, hydrophobic monomers with different alkyl chain lengths have a significant influence on the energy dissipated by hydrogels. Dissipated energy was an indicator to analyze the toughness of the hydrogels. Fig. 7 shows the hysteresis loops and dissipated energy condition for the four hydrogels with different hydrophobic segments at a strain of $1000 \%$. The P(AAm/LMA)-LP and P(AAm/HDMA)-LP hydrogels displayed prominent hysteresis loops, but inconspicuous hysteresis loops were exhibited for P(AAm/MMA)-LP and $\mathrm{P}(\mathrm{AAm} / \mathrm{HMA})$-LP hydrogels. Accordingly, the dissipated energy of the P(AAm/LMA)-LP hydrogel and P(AAm/HDMA)-LP hydrogel was up to 129.7 and $107.5 \mathrm{KJ} \mathrm{m}^{-3}$, which were much higher than those of the P(AAm/MMA)-LP hydrogel $\left(53.24 \mathrm{~kJ} \mathrm{~m}^{-3}\right)$ and $\mathrm{P}(\mathrm{AAm} / \mathrm{HMA})-\mathrm{LP}$ hydrogel $\left(65.38 \mathrm{~kJ} \mathrm{~m}^{-3}\right)$. The results indicated that the disentanglement between LPs and longer alkyl chains could effectively dissipate a great amount of energy under the external force; this endowed the hydrogels with excellent toughness.

Furthermore, the successive loading-unloading test was carried out to evaluate the mechanical properties of hydrogels in response to the change of alkyl chain length (Fig. 8). For the four hydrogel samples, although a significant hysteresis occurred in the first loading-unloading curve, a smaller hysteresis was observed in the second to fifth cycle, and they followed the path of the first unloading curve. The results indicated that unrecovered damage occurred in the first curve that generated a permanent residual elongation..$^{30}$ Especially, the stress of hydrogels with MMA and HMA slightly increased. This was ascribed to the fact that the short chains disentangled from the LPs in the first loading process and rearranged in the following stretching process; this led to the slight improvement of stress.

The swelling characteristic is one of the important factors for studying the properties of hydrogels. Fig. 9(a) shows the swelling behavior of hydrogels containing different hydrophobic segments. Obviously, the P(AAm/MMA)-LP and P(AAm/ HMA)-LP hydrogels collapsed after being soaked in water for $24 \mathrm{~h}$. The swelling ratio of the P(AAm/LMA)-LP and P(AAm/ HDMA)-LP hydrogels at the swelling equilibrium state was about 17.2 and 38.6, respectively. As previously discussed, the entanglement between the LPs and shorter alkyl chains was loose, and the internal network structure of the hydrogels was unstable; this resulted in the destruction of the hydrogels during the process of swelling. On the contrary, the longer
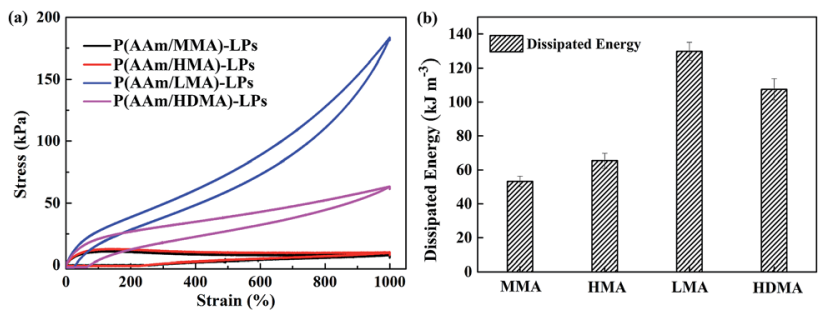

Fig. 7 (a) Loading-unloading curves and (b) dissipated energy of hydrogels with MMA, HMA, LMA, and HDMA alkyl chains at a strain of $1000 \%$.
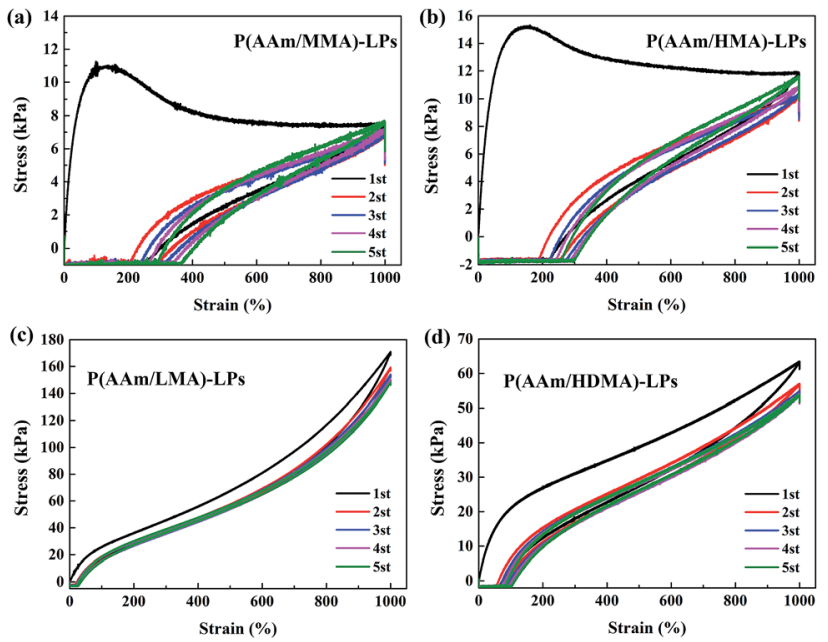

Fig. 8 The successive loading-unloading tests of (a) P(AAm/MMA)LPs, (b) P(AAm/HMA)-LPs, (c) P(AAm/LMA)-LPs, and (d) P(AAm/ HDMA)-LPs.

hydrophobic chains of LMA and HDMA were difficult to disentangle from LPs in the swelling process and could maintain the network of the hydrogels; therefore, the corresponding

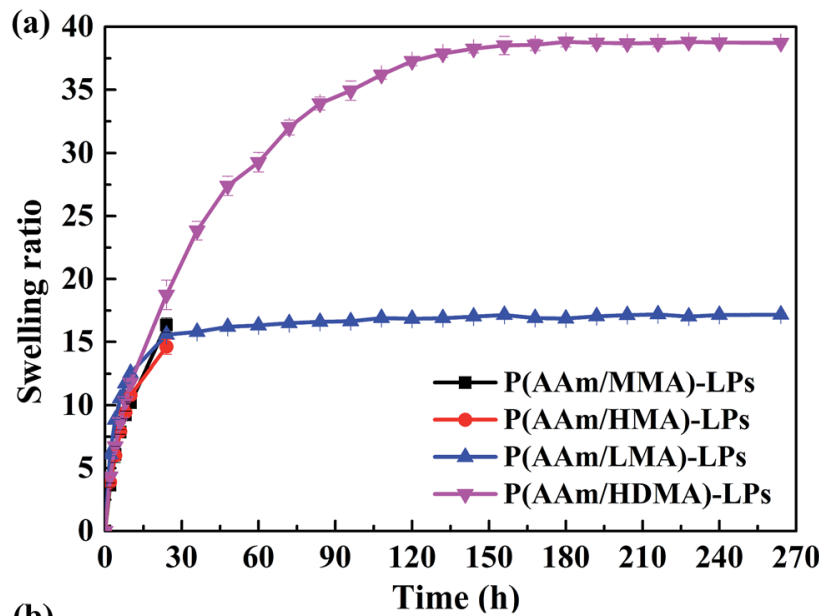

(b)

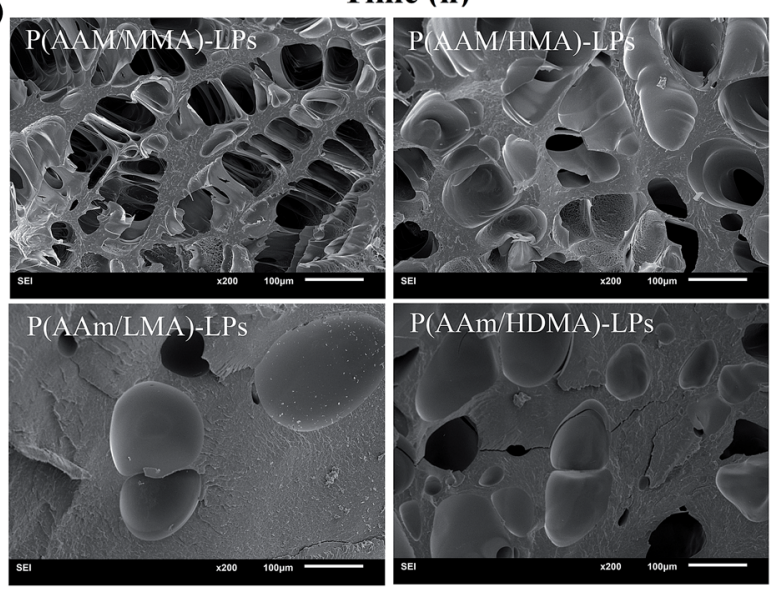

Fig. 9 (a) Swelling behavior and (b) SEM images of the hydrogels with hydrophobic segments of MMA, HMA, LMA, and HDMA. 
hydrogels could keep the original shape without fracture. The swelling ratio was related to the cross-linking density of the hydrogels. As shown in the SEM image (Fig. 9(b)), the crosslinking density of $\mathrm{P}$ (AAm/LMA)-LP and P(AAm/HDMA)-LP hydrogels was higher than that of $\mathrm{P}(\mathrm{AAm} / \mathrm{MMA})-\mathrm{LP}$ and P(AAm/HMA)-LP hydrogels; this was consistent with the swelling result. The higher cross-linking density would lead to a lower swelling degree.

The content of LPs has a great influence on the swelling ratio of the $\mathrm{P}(\mathrm{AAm} / \mathrm{LMA})-\mathrm{LP}$ hydrogels. Fig. 10 shows that the swelling ratio quickly increased at the beginning and then gradually reached a steady value. When the content of LPs varied from 0 to $0.5 \mathrm{wt} \%$, the swelling ratio decreased significantly and reached the minimum value at the concentration of $0.5 \mathrm{wt} \%$. This is because the introduction of LPs changed the cross-linking structure of the hydrophobic association hydrogels. The homogeneity and the effective cross-linking density of the hydrogel network will significantly increase with the increase of LP concentration; therefore, the hydrogels exhibited the lowest swelling ratio at the LP concentration of $0.5 \mathrm{wt} \%$. Furthermore, the hydrogels with $0.5 \mathrm{wt} \%$ LPs exhibited the best tensile strength. However, the swelling ratio increased with the increase in the LP contents from 0.5 to $1.5 \mathrm{wt} \%$. A possible reason was that the excessive LPs resulted in the heterogeneity of the hydrogel network structure and the effective cross-linking density reduced; hence, the swelling ratio of the hydrogels increased.

The kinetics of the copolymerization process was monitored by rheological experiments, as shown in Fig. 11(a-e). It was clearly observed from the gelation profile that a certain induction period existed in the four hydrogel samples. The gelation time of polyacrylamide with MMA, HDMA, LMA, and HDMA was $409,347,480$, and $614 \mathrm{~s}$, respectively. The gelation time first decreased and then increased with the increase of the alkyl chain length owing to the different hydrophobicities and steric hindrances of the hydrophobic monomers. The steric hindrance increased because the alkyl chain length increased, such that the gelation time increased from HMA to HDMA.

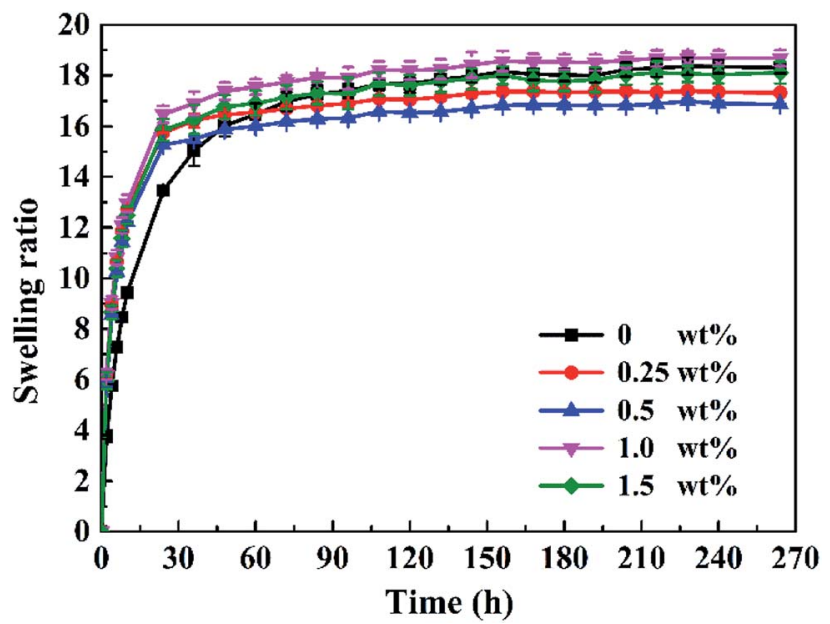

Fig. 10 The influence of the LP content on the swelling ratio of P(AAm/LMA)-LP hydrogels.
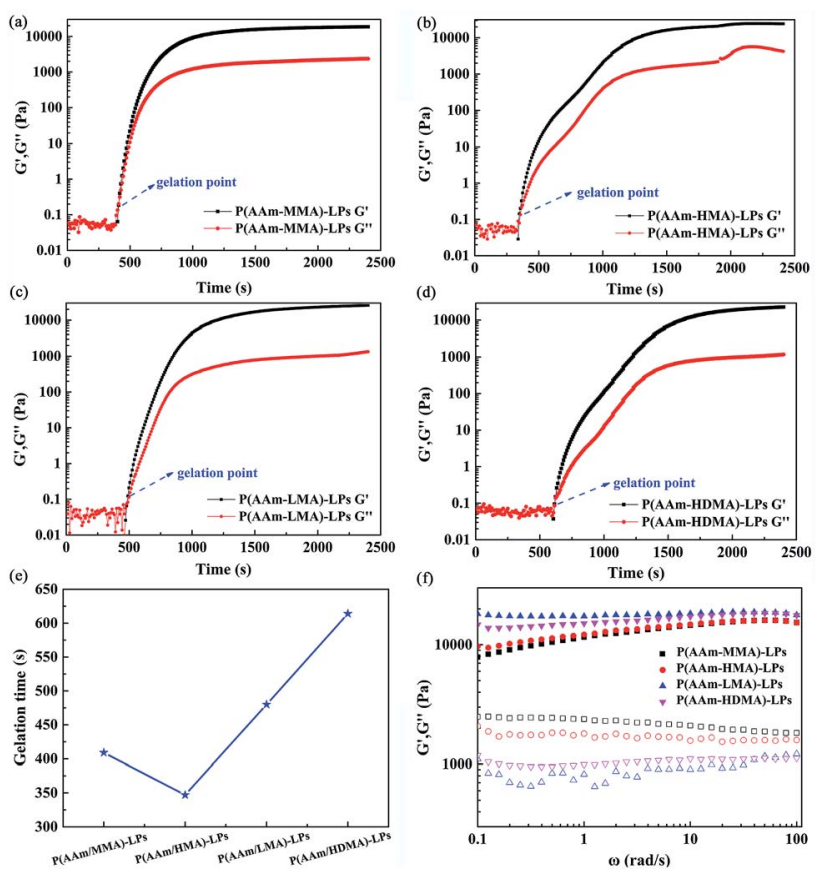

Fig. 11 Storage modulus $\left(G^{\prime}\right)$ and loss modulus $\left(G^{\prime \prime}\right)$ shown as a function of reaction time during the copolymerization of AAm with (a) MMA, (b) HMA, (c) LMA, and (d) HDMA. (e) The gelation time for four different hydrogels. (f) Storage modulus $\left(G^{\prime}\right)$ (filled symbols) and loss modulus $\left(G^{\prime \prime}\right)$ (open symbols) of hydrogels shown as a function of the frequency.

However, the gelation time of AAm and MMA was longer than that for HMA. This was attributed to the fact that the hydrophobicity of MMA was weaker than that of HMA. Simultaneously, it was found that AAm and HMA formed a gel more easily possibly due to appropriate their hydrophobicity and steric hindrance. Then, both the storage modulus $G^{\prime}$ and loss modulus $G^{\prime \prime}$ of the hydrogel samples increased sharply with the increase of reaction time and reached a plateau value. The copolymerization process was considered to be completed for the system.

The frequency sweeping test was carried out at the frequencies varying from 0.01 to $100 \mathrm{rad} \mathrm{s}^{-1}$. As shown in Fig. 11(f), the $\mathrm{P}(\mathrm{AAm} / \mathrm{MMA})-\mathrm{LP}$ and $\mathrm{P}(\mathrm{AAm} / \mathrm{HMA})-\mathrm{LP}$ hydrogels exhibited a viscoelastic behavior. As expected, both the storage modulus $G^{\prime}$ and loss modulus $G^{\prime \prime}$ are dependent on frequency. With the increase of frequency, the storage modulus $G^{\prime}$ increased, but the loss modulus $G^{\prime \prime}$ decreased. This is characteristic of viscoelastic materials. However, the P(AAm/LMA)-LP and P(AAm/HDMA)-LP hydrogels exhibited a gel-like behavior. The storage modulus $G^{\prime}$ is higher than the loss modulus $G^{\prime \prime}$ in the abovementioned frequency range, and both are nearly independent of frequency. Moreover, note that the $G^{\prime}$ curves of all hydrogels are smooth, but the $G^{\prime \prime}$ curves are relatively rough. Obviously, this feature was similar to that of the elastic materials.

\section{Conclusions}

A series of hydrogels with different long alkyl chains were successfully fabricated by introducing LPs as hydrophobic 
association centers. LPs played a significant role in improving the mechanical strength of the hydrogels, and methacrylate could adsorb on the surface of LPs due to hydrophobic interactions. The mechanical properties of the LP-Gel could be tuned by adjusting the concentration of LPs and the length of alkyl chains of hydrophobic monomers. The degree of hydrophobic interaction was different between methacrylate and the LPs; this caused the hydrogels to display different mechanical strengths and swelling ratios. The optimal P(AAm/LMA)-LP hydrogel possessed a high fracture of $1.2 \mathrm{MPa}$ and a large elongation of $2336 \%$. Therefore, the current study may provide a new thought and theoretical support for the design of a novel latex particle hydrogel and further expand the applications of hydrogels.

\section{Conflicts of interest}

There are no conflicts to declare.

\section{Acknowledgements}

This research was supported by a grant received from the National Natural Science Foundation of China (NSFC) (No. 51473023 and 51103014), Science and Technology Department of Jilin Province (No. 20150204064G), Education Department of Jilin Province (No. 201582), and Changchun Municipal Science and Technology Bureau (No. 14KG054).

\section{Notes and references}

1 S. Hong, D. Sycks, H. F. Chan, S. Lin, G. P. Lopez, F. Guilak,

K. W. Leong and X. Zhao, Adv. Mater., 2015, 27, 4035.

2 D. E. Discher, D. J. Mooney and P. W. Zandstra, Science, 2009, 324, 1673.

3 L. Dong, A. K. Agarwal, D. J. Beebe and H. Jiang, Nature, 2006, 442, 551.

4 J. Yan, V. A. Pedrosa, A. L. Simonian and A. Revzin, ACS Appl. Mater. Interfaces, 2010, 2, 748.

5 E. Wang, M. S. Desai and S. W. Lee, Nano Lett., 2013, 13, 2826.

6 J. Duan, X. Liang, K. Zhu, J. Guo and L. Zhang, Soft Matter, 2016, 13, 345.

7 B. Xue, M. Qin, T. Wang, J. Wu, D. Luo, Q. Jiang, Y. Li, Y. Cao and W. Wang, Adv. Funct. Mater., 2016, 26, 9053.

8 Y. Okumura and K. Ito, Adv. Mater., 2001, 13, 485.

9 C. Katsuno, A. Konda, K. Urayama, T. Takigawa, M. Kidowaki and K. Ito, Adv. Mater., 2013, 25, 4636.

10 A. Bin Imran, K. Esaki, H. Gotoh, T. Seki, K. Ito, Y. Sakai and Y. Takeoka, Nat. Commun., 2014, 5, 5124.
11 K. Haraguchi and T. Takehisa, Adv. Mater., 2002, 14, 1120.

12 G. Gao, G. Du, Y. Sun and J. Fu, ACS Appl. Mater. Interfaces, 2015, 7, 5029.

13 D. Wu, M. Yi, H. Duan, J. Xu and Q. Wang, Carbon, 2016, 108, 394.

14 S. Pacelli, P. Paolicelli, G. Moretti, S. Petralito, S. Di Giacomo, A. Vitalone and M. A. Casadei, Eur. Polym. J., 2016, 77, 114.

15 J. P. Gong, Y. Katsuyama, T. Kurokawa and Y. Osada, Adv. Mater., 2003, 15, 1155.

16 Q. Chen, L. Zhu, H. Chen, H. Yan, L. Huang, J. Yang and J. Zheng, Adv. Funct. Mater., 2015, 25, 1598.

17 J. P. Gong, Soft Matter, 2010, 6, 2583.

18 Q. Chen, L. Zhu, C. Zhao, Q. Wang and J. Zheng, Adv. Mater., 2013, 25, 4171.

19 T. Huang, H. G. Xu, K. X. Jiao, L. P. Zhu, H. R. Brown and H. L. Wang, Adv. Mater., 2007, 19, 1622.

20 C. He, K. Jiao, X. Zhang, M. Xiang, Z. Li and H. Wang, Soft Matter, 2011, 7, 2943.

21 T. Cai, G. Wang, S. Thompson, M. Marquez and Z. Hu, Macromolecules, 2008, 41, 9508.

22 Y. Tan, K. Xu, P. Wang, W. Li, S. Sun and L. Dong, Soft Matter, 2010, 6, 1467.

23 J. Zhao, K. Jiao, J. Yang, C. He and H. Wang, Polymer, 2013, 54, 1596.

24 G. Jiang, C. Liu, X. Liu, G. Zhang, M. Yang and F. Liu, Macromol. Mater. Eng., 2009, 294, 815.

25 G. Jiang, C. Liu, X. Liu, Q. Chen, G. Zhang, M. Yang and F. Liu, Polymer, 2010, 51, 1507.

26 S. Abdurrahmanoglu, M. Cilingir and O. Okay, Polymer, 2011, 52, 694.

27 C. Bilici, V. Can, U. Nöchel, M. Behl, A. Lendlein and O. Okay, Macromolecules, 2016, 49, 7442.

28 T. Zhao, M. Tan, Y. Cui, C. Deng, H. Huang and M. Guo, Polym. Chem., 2014, 5, 4965.

29 S. Abdurrahmanoglu, V. Can and O. Okay, Polymer, 2009, 50, 5449.

30 D. C. Tuncaboylu, A. Argun, M. Sahin, M. Sari and O. Okay, Polymer, 2012, 53, 5513.

31 T. T. Gao, N. Niu, Y. D. Liu, X. L. Liu, G. Gao and F. Q. Liu, RSC Adv., 2016, 6, 43463.

32 W. Li, H. An, Y. Tan, C. Lu, C. Liu, P. Li, K. Xu and P. Wang, Soft Matter, 2012, 8, 5078.

33 W. Cui, J. Ji, Y.-F. Cai, H. Li and R. Ran, J. Mater. Chem. A, 2015, 3, 17445.

34 X. Y. Ren, Z. Yu, B. Liu, X. J. Liu, Y. J. Wang, Q. Su and G. H. Gao, RSC Adv., 2016, 6, 8956. 